\title{
Accuracy Enhancement by Selective Use of Branch History in Embedded Processor
}

\author{
Jong Wook Kwak ${ }^{1}$, Seong Tae Jhang ${ }^{2}$, and Chu Shik Jhon ${ }^{1}$ \\ ${ }^{1}$ Department of Electrical Engineering and Computer Science, \\ Seoul National University, Shilim-dong, Kwanak-gu, Seoul, Korea \\ \{leoniss, csjhon\}@panda.snu.ac.kr \\ ${ }^{2}$ Department of Computer Science, The University of Suwon, \\ Suwon, Gyeonggi-do, Korea \\ stjhang@suwon.ac.kr
}

\begin{abstract}
The branch prediction accuracy is one of essential parts of performance improvement in embedded processors as well as modern microarchitectures. Until now, the length of branch history has been statically fixed for all branch instructions, and the history length is usually selected in accordance with the size of prediction table. In this paper, we propose a dynamic per-branch history length adjustment policy, which can dynamically change the history length for each branch instruction. The proposed solution tracks data dependencies of branch instructions and identifies strongly correlated branches in branch history. Compared to the previous bimodal style predictors and the fixed history length predictors in embedded processors, our method provides better history length for each branch instruction, resulting in substantial improvement in prediction accuracy.
\end{abstract}

Keywords: Branch Prediction, Branch History, History Length Adjustment, Data Dependency, gshare Predictor.

\section{Introduction}

To achieve higher performance, recent microarchitectures have made use of deeper pipeline, dynamic scheduling and multi-issue superscalar processor technologies. In the field of embedded processors, such technology trends are expected to be realized in a near future as well. Consequently, accurate branch predictor will be one of essential parts of modern embedded processors, because the penalty for a branch miss-prediction increases as the number of pipeline stages and the number of instructions issued per cycle increase [1]. Although there have been many proposals of complex hybrid branch predictors in modern microarchitectures, they can not directly be implemented in embedded processors, due to the strict hardware resource constraints in their environments. Instead, most branch predictors in embedded processors have used the small-scale bimodal style predictors[2]. In bimodal predictors, the only information in branch prediction is the address of branch instruction(PC), without Global Branch History(GBH). 
However, as shown in many previous works, branch instructions have a special feature in their executions, called correlation, and the prediction accuracy is expected to increase if the predictor additionally utilizes the correlation features of branch instructions[3][4]. In this paper, we propose a new novel algorithm and hardware structure, which can be implemented in embedded processors with providing the correlation features, as the alternative of bimodal predictors in embedded processors. Our policy is much more profitable to high-performance embedded systems. The rest of this paper is organized as follows. Section 2 describes the backgrounds and the related works about this paper. Section 3 proposes dynamic per-branch history length adjustment and explains its distinctive algorithm and required hardware structure. In section 4 , we simulate our proposal and discuss the simulation results. Finally, section 5 concludes this paper.

\section{Backgrounds and Related Works}

Various vectors of information can be used as an input of branch predictors. The branch address was firstly proposed to index the Pattern History Table (PHT). This style of predictors is often called as the bimodal predictor and it is still widely used in current microarchitectures, because the bimodal distribution of branch execution is one of main characteristics of most branch instructions [5]. Further, in the field of embedded environments, bimodal predictor is an essential branch predictor. For examples, XScale Processor implements the bimodal predictor with 2 bit saturation counter in Branch Target Buffer(BTB)[2]. In the family of ARM processors, they implement simple bimodal style branch predictors as well, due to the strict hardware resource constraints[6]. However, it has been shown that some branches, especially conditional branches, are strongly correlated to the outcomes of a few past branch instructions. The 2-level adaptive branch predictor and its variations utilize this idea. This mechanism uses two levels of branch history to make the predictions; the history of the last $k$ branches encountered and the branch behavior for the last $s$ occurrences of the specific pattern of these $k$ branches[3].

Meanwhile, the length of branch history is statically fixed for all branch instructions, and the history length is usually selected in accordance with the size of PHT. However, as shown in previous works, different branch instructions require different length histories to achieve high prediction accuracies [7][8][9]. In previous works, [7] and [8] require prior-profiling to change the history length. Although [9] provides dynamic history length fitting method, it also requires many intervals which consist of a fixed number of consecutive branch instructions, to profile and change the history length, thus it is semi-dynamic. On the whole, these intervals induce the overall decrease of IPC. However, our policy adjusts the history length in program execution time, that is fully-dynamic. Moreover, our policy is per-branch method and provides optimal history length for each branch instruction, which is not the case of previous works. 


\section{History Length Adjustment in Branch Prediction}

In this section, we propose Dynamic Per-Branch History Length Adjustment (called DpBHLA Policy), and additionally we show its algorithm and required hardware structure. The proposed solution tracks data dependencies of branch instructions and identifies strongly correlated branches(called key branch) in branch history, based on operand register in branch instruction. By identifying the key branch, the DpBHLA policy selectively uses the information of key branch in $\mathrm{GBH}$, resulting in different history length for each branch instruction.

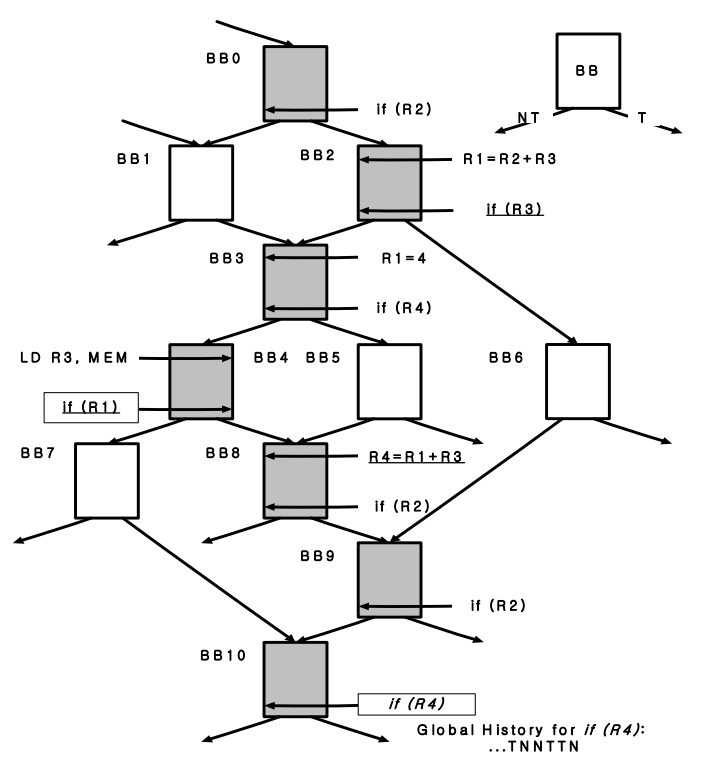

Fig. 1. Example Scenario

Figure 1 shows the example scenario to illustrate the DpBHLA mechanism. Figure 1 is a kind of Control Flow Gragh(CFG), composed of Basic Blocks $(\mathrm{BBs})$. Each basic block consists of one branch instruction and a few other instructions which do not change the execution path. if $\left(R_{4}\right)$ in BB10 is the branch instruction in which we want to predict the execution path. The GBH for if $\left(R_{4}\right)$ in BB10 is currently (...TNNTTN). To identify the key branch, we introduce Branch Register Dependency Table (Br_RDT), which stores the key branch information. Figure 2 shows its structure. In Figure 2, the number of entries in Br_RDT is the same as the number of architectural registers and the width of each entry is the same as the history length $n$, according to the $2^{n} \mathrm{PHT}$ size. To select the optimal history length for each branch instruction, each entry in Br_RDT is handled by the following algorithm, shown in Figure 3.

The algorithm in Figure 3 mainly controls each entry of Br_RDT, based on the instruction format. The important instruction formats in our algorithm are 


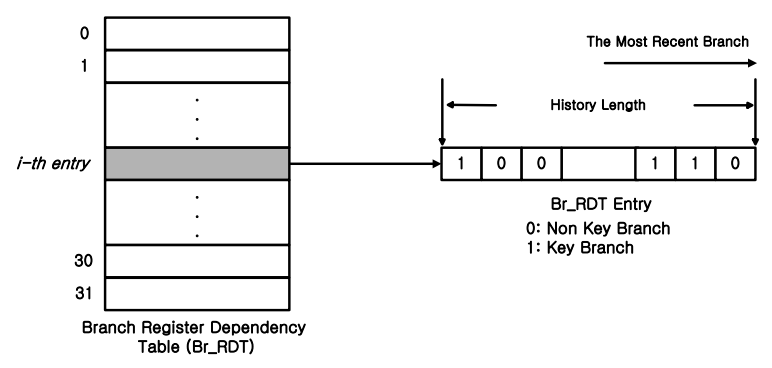

Fig. 2. Branch Register Dependency Table

(1) conditional branch instruction and (2) register writing instruction. In case of conditional branch instructions, the Length_Indicator takes the entries of source operands in branch instructions, and newly set the value of logic 1 into the LSB field of $R e g_{-} S r c_{i}$ in Br_RDT, with shifting one bit left in all Br_RDT entry. In case of register writing instructions, each $R e g_{-} S r c_{i}$ in Br_RDT is bit-wise-ored, and the entry of Br_RDT(Reg_dest) takes the result.

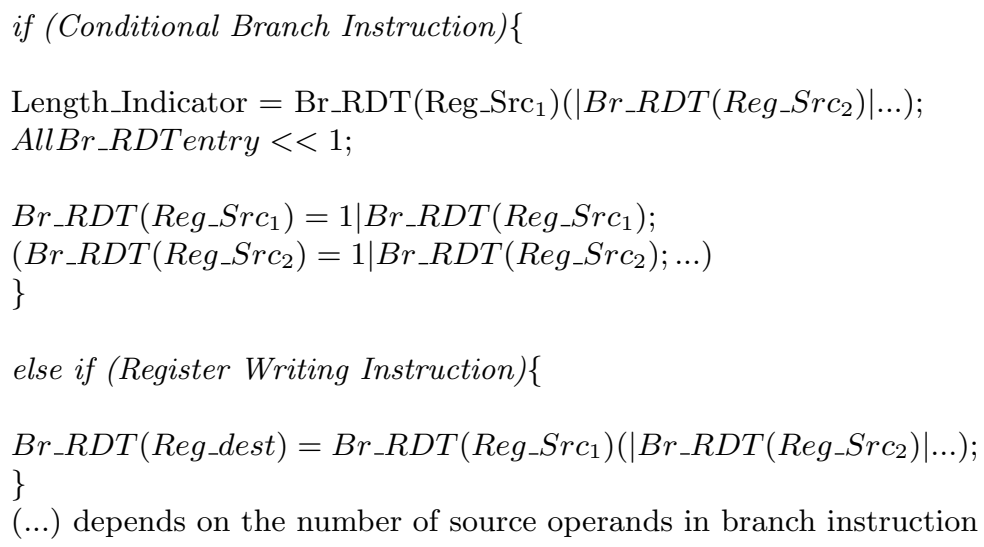

Fig. 3. History Length Adjustment Algorithm

Figure 4 illustrates the example contents for selecting the history length of if $\left(R_{4}\right)$ in BB10. In Figure 4, the entry of R4 in BB10 is (...0000100) and the Length_Indicator uses the entry of R4 in Br_RDT at the end of the algorithm. The entry of Br_RDT indicates whether $i$-th field of each Br_RDT entry is BB which contains the key branch or not. Therefore, this vector describes that the BB which has the data dependency with R4 in BB10 is 3 ahead BB (i.e., third field) from BB10, and it additionally indicates that the strongly correlated branch (i.e., key branch) with if $\left(R_{4}\right)$ in BB10 exists in 3 ahead $B B$ (i.e., BB4). Actually, R4 is affected by the value of $\mathrm{R} 1$ and $\mathrm{R} 3$ in $\mathrm{BB} 8$ 

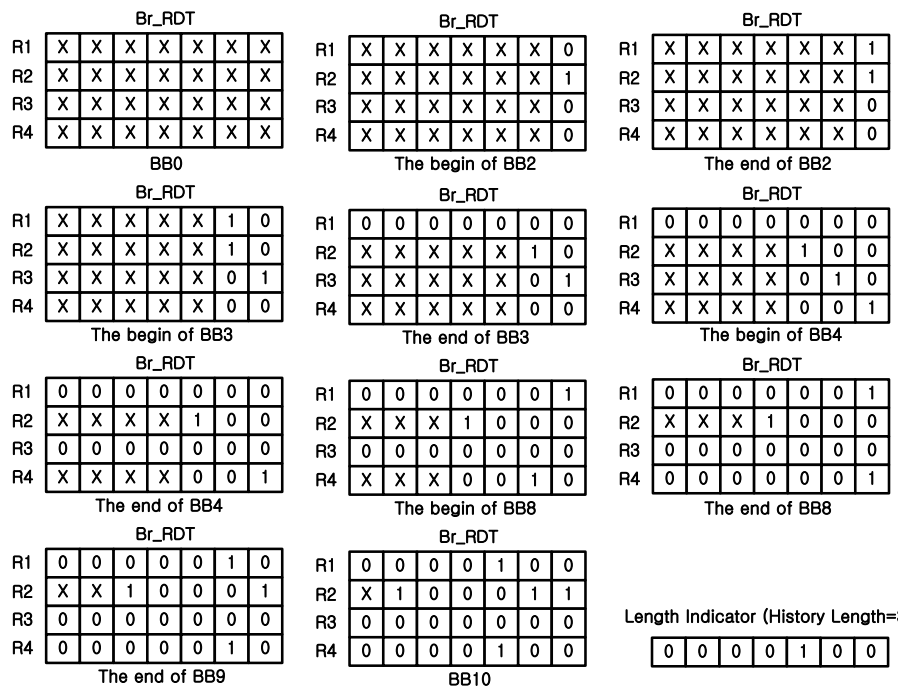

Br_RDT
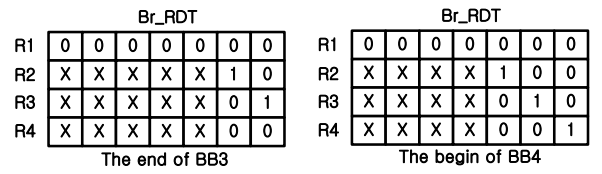

Br_RDT

Br_RDT
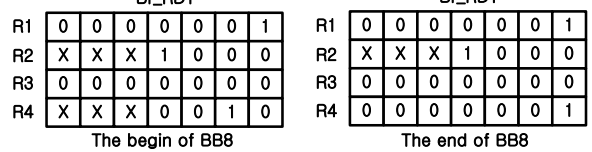

Br_RDT

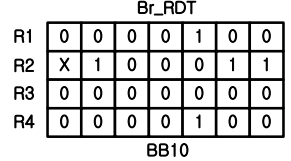

Length Indicator (History Length $=3$ )

\begin{tabular}{|l|l|l|l|l|l|l|}
\hline 0 & 0 & 0 & 0 & 1 & 0 & 0 \\
\hline
\end{tabular}

Fig. 4. The Example Contents of Br_RDT

$(R 4=R 1+R 3)$, and the value of $\mathrm{R} 1$ and $\mathrm{R} 3$ is used by BB4 (if $(R 1))$ and BB2 $(i f(R 3))$ respectively. By the or -ing function of the algorithm $\left(B r_{-} R D T(R 4)=\right.$ $\left.B r_{-} R D T(R 1) \| B r_{-} R D T(R 3)\right), \mathrm{R} 4$ in Br_RDT has the value of (...0000001) at the end of BB8. In this way, R4 has (...0000100) at the end of the algorithm. This result indicates that the key branch for if $\left(R_{4}\right)$ in BB10 is if $(R 1)$ in BB4. Consequently, when predicting if $\left(R_{4}\right)$ in BB10, we use history length 3 (TTN, i.e., BB4, BB8 and BB9), instead of predefined static history length $n$ for the $2^{n}$ PHT size(...TNNTTN). As shown in this example, by identifying the key branch, the different history length can be used for each branch instruction.

\section{Performance Evaluation}

In this section, we evaluate the performance of our proposal. We use an eventdriven simulator, SimpleScalar[10], for our simulation. As benchmark programs, we use SPEC 2000 application suits[11].

At first, Figure 5 shows miss-prediction rate(\%) vs. history length, for each application. In Figure 5, we vary the number of PHT entries from 512 to 4K, and we change the history length from 1 to $n$ for each $2^{n}$ PHT size. Although the results are strongly application dependent, the selected four simulation results show the representative result patterns, compared to other applications. As shown in Figure 5, the prediction accuracy is significantly affected by the history length. For example, the difference between the best accuracy and the worst accuracy is $2.1 \%$, in case of $181 . m c f$ in 512 PHT. Furthermore, the best history length is also dependent on the PHT size, as shown in 181.mcf: (1) history length 6 in 512 PHT vs. (2) history length 8 in 4K PHT. Overall, these 
results indicate that the optimal history length is truly application and system dependent. Therefore, we should use the dynamic method to optimally select the best history length, regardless of applications and system characteristics.

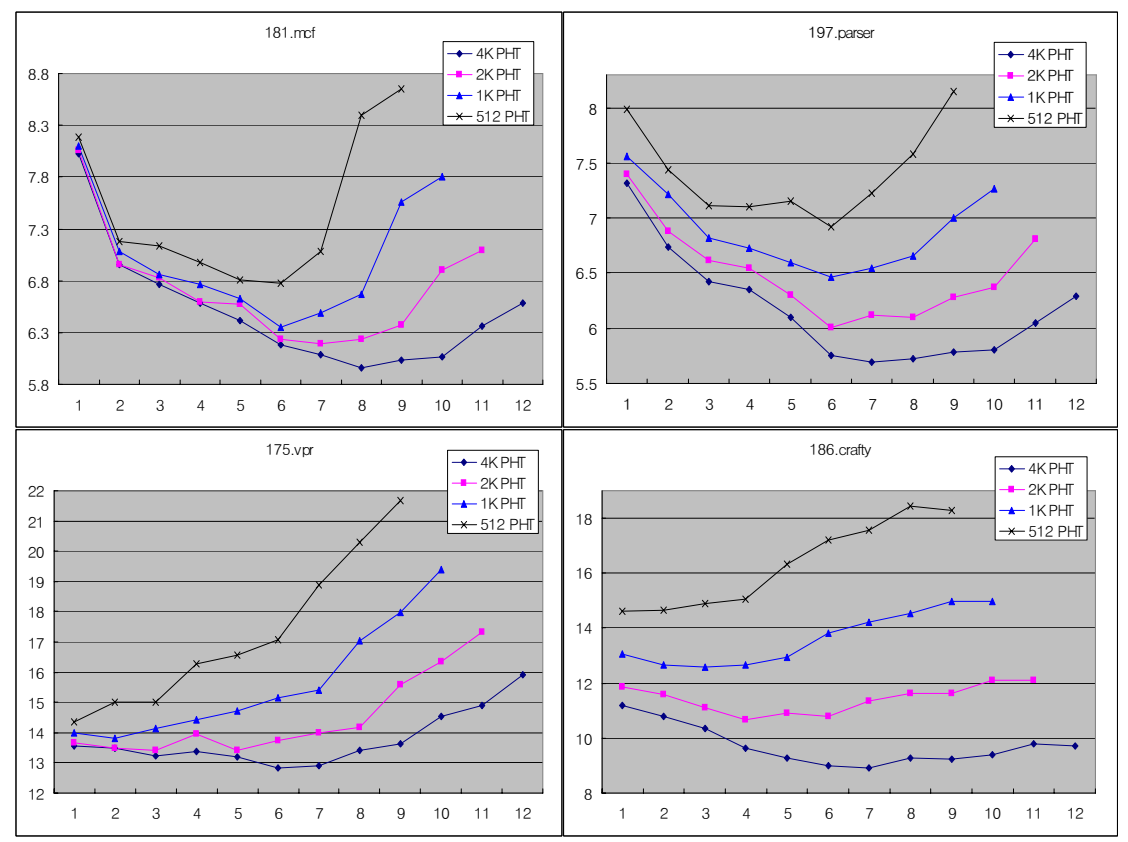

Fig. 5. Branch Miss-Prediction Rate vs. History Length

On the other hand, Figure 6 shows the branch prediction accuracy(\%) vs. PHT size. Generally, it has been known that the simple bimodal predictor is superior to the 2-level predictor or the gshare predictor in case of small PHT size, because a short length of the GBH can not provide enough information to distinguish each branch instruction. Our simulation shows this phenomenon as well, in case of simulation results in 512 PHT entries, as shown in average result. Due to this reason, the bimodal predictor is usually adopted by the small-scale embedded processors. Besides, as the PHT size increases, the 2-level predictor and the gshare predictor, on the contrary, provide better prediction accuracy than the bimodal predictor.

Figure 7 shows the miss-prediction rate(\%) of DpBHLA policy in 512, 1K, and $2 \mathrm{~K}$ PHT, respectively. In our simulation, Fixed Length depicts the result of conventional fixed and static history length policy, and Length Adjustment is our proposal. As shown in Figure 7, DpBHLA policy outperforms Fixed Length policy, $2.1 \%$ in average, and the improvement is up to $4.7 \%$ in case of $175 . v p r$. Moreover, in small-scale system environment(such as 512 PHT), DpBHLA significantly outperforms Fixed Length policy, and it even outperforms the bimodal 

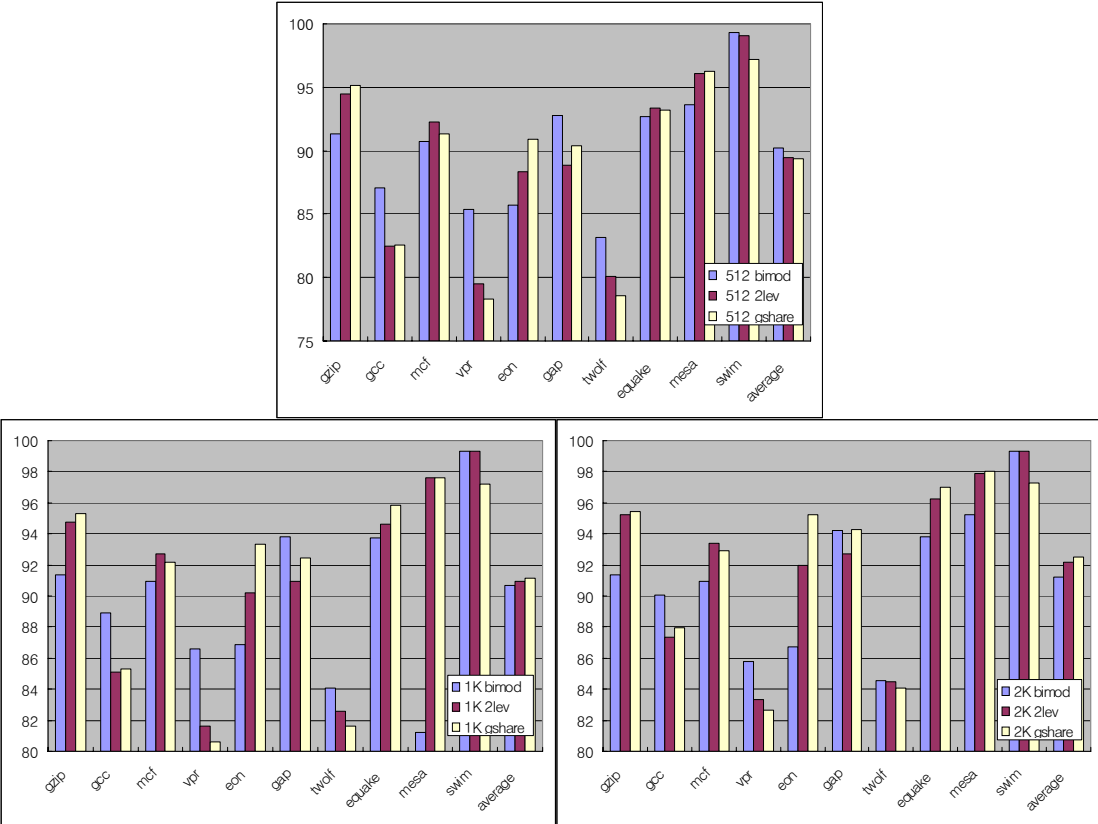

Fig. 6. Branch Prediction Accuracy vs. PHT Size
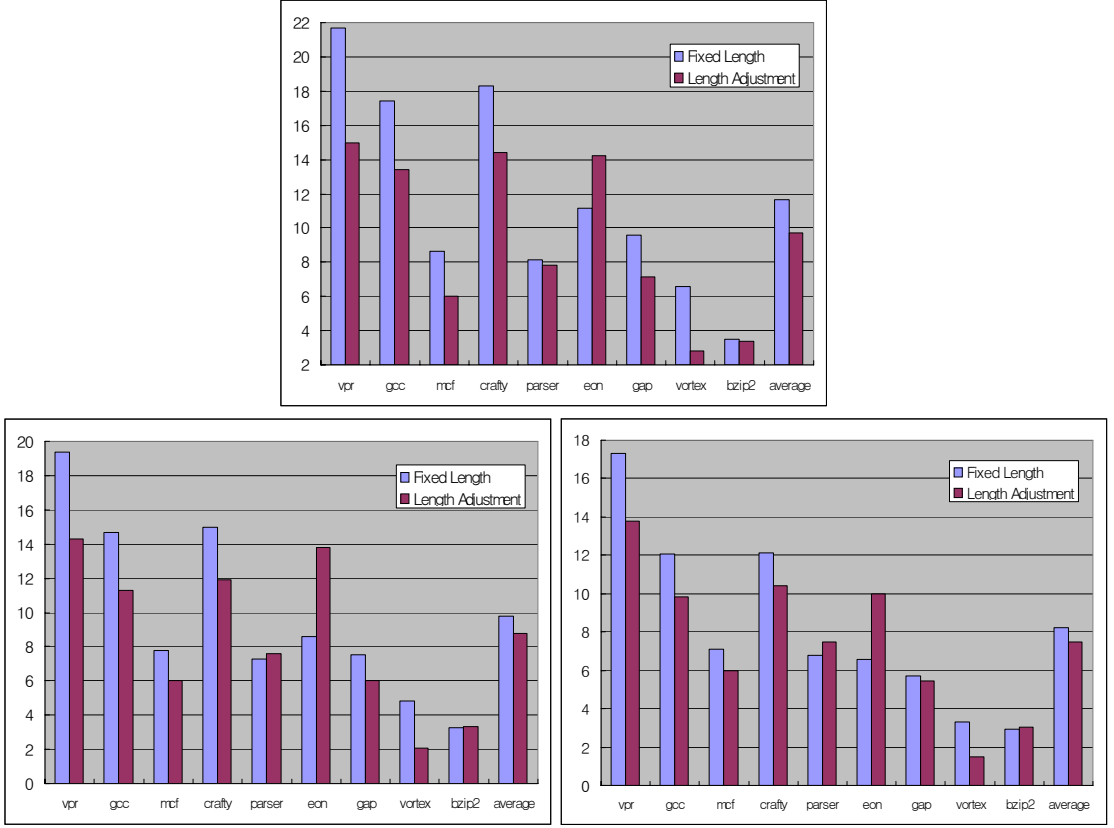

Fig. 7. The Reduction of Branch Miss-Prediction in DpBHLA Policy 
predictor which usually provides better prediction accuracies in small embedded systems. This result indicates that the DpBHLA policy can be implemented in the microarchitectures which use the previous fixed history length policy. Further, the DpBHLA policy can also be implemented in small-scale embedded processors, with better prediction accuracy than the bimodal predictors.

\section{Conclusion}

To realize the performance potential of today's widely-issued, deeply-pipelined processor environments, the accurate branch predictor becomes one of essential parts of modern microarchitectures and embedded processors. In this paper, we presented Dynamic Per-Branch History Length Adjustment (DpBHLA) policy, which is fully-dynamic and per-branch method to efficiently select the history length, regardless of applications and system characteristics. The proposed solution provided up to $4.7 \%$ improvement in prediction accuracy, compared to the fixed history length policy, and it even outperformed bimodal predictors in small system environments.

\section{References}

1. J. L. Hennessy and D.A Patterson, "Computer Architecture : A Quantitative Approach", Third Edition, Morgan Kaufmann Publishers, Inc, 2001.

2. Intel XScale Core Developer's Manual, January, 2004

3. Yeh, T. Y. and Patt, Y. N., "Two-level adaptive branch prediction", In Proceedings of the 24th ACM/IEEE International Symposium on Microarchitecture, 51-61, 1991

4. McFarling, S., "Combining branch predictors. Tech. Rep. TN-36m", Digital Western Research Lab., June, 1993

5. Keith Diefendorff, "K7 Challenges Intel, New AMD Processor Could Beat Intel's Katmai", Microprocessor Report, Vol. 12, No. 14, 1998

6. Steve Furber, "ARM System-on-Chip Architecture", 2nd edition, Addison-Wesley, 2000

7. J. Stark, M. Evers, and Y. N. Patt, "Variable length path branch prediction", In Proc. 8th Int'l Conf. on Architectural Support for Programming Languages and Operating Systems, pp. 170-179, 1998.

8. M.-D. Tarlescu, K. B. Theobald, and G. R. Gao, "Elastic history buffer: A low-cost method to improve branch prediction accuracy", In Proc. Int'l Conf. on Computer Design, pp. 82-87, 1997.

9. T.Juan, S. Sanjeevan, and J. J. Navarro, "Dynamic history length fitting: A third level of adaptivity for branch prediction", In Proc. 25th Int'l Symp. on Computer Architecture, pp. 155-166, 1998.

10. D. Burger, T. M. Austin, and S. Bennett, "Evaluating future micro-processors: the SimpleScalar tool set", Tech. Report TR-1308, Univ. of Wisconsin-Madison Computer Sciences Dept., 1997

11. SPEC CPU2000 Benchmarks, http://www.specbench.org 